Table 1 PTU-021

\begin{tabular}{cllccll}
\hline Location & \multicolumn{7}{l}{ Duke's Stage } \\
\hline Right & Left & A & B & C1 & C2 & D \\
3 & 23 & 13 & 6 & 5 & 1 & 1 \\
$11.5 \%$ & $88.5 \%$ & $50 \%$ & $23 \%$ & $19 \%$ & $4 \%$ & $4 \%$ \\
\hline
\end{tabular}

was 65 (range 62-72). The site and stage of the lesion detected is shown in the table below.

$73 \%$ of cancers identified were Dukes stage A/B, 27\% were C/D. Most lesions were left sided (88.5\%).

Conclusion Accurate and detailed databases set up by the National BCSP have the potential to facilitate further research into better understanding the rate of development of bowel cancer and possibly enable improvements in screening methods in the future to ensure that greater numbers of cancers are detected early. In this cohort the lesions were predominantly in the left colon and at an early stage. The reasons for a negative $\mathrm{FOB}$ two years earlier could be the consequence of intermittent blood loss or possibly inadequate haemolysis which is necessary for the FOBt kit to detect the blood. This is a known limitation of the FOBt. Our data supports the use of flexible sigmoidoscopy as an adjunct to the present screening program. Funding for this was recently announced by the Coalition Government following recommendations by the National Screening committee. This data also suggests that the presently used FOBt algorithm needs national audit.

Competing interests None.

Keywords bowel cancer screening, faecal occult blood.

\section{PTU-021 $\downarrow$ COLORECTAL CANCER IN FIRST ROUND FOB NEGATIVE, 2ND ROUND FOB POSITIVE BOWEL CANCER SCREENING SUBJECTS: TUMOUR SITE AND STAGE}

doi:10.1136/gut.2011.239301.149

P Patel, T Pirani, M Bouwe, R Leicester, A Poullis Endoscopy Department, St. George's Hospital, London, UK

Introduction The NHS bowel cancer screening program (NHS BCSP) currently screens persons between the ages of 60 and 69 for bowel cancer (in the process of being extended to age 74). The aim is to detect early cancer and reduce mortality associated with CRC. The NHS BCSP began in 2006 in England and Nationwide coverage was achieved in 2010. Individuals are invited biennially to provide a stool sample for faecal occult blood test (FOBt) analysis. South West London BCS centre at St. George's Hospital has now completed 2 screening rounds in our screen population. We determined the cancer site and stage detected in patients who had positive FOBt during the second screening round, but negative FOBt at the first screening round two years earlier.

Methods Data from patients with a diagnosis of colorectal cancer in the second round was examined. Patients who had a negative FOBt in round 1 but a positive FOBT in the 2 nd round were selected. Staging imaging and histology was reviewed to highlight the location and stage of cancer detected.

Results 26 patients with CRC and a negative FOBt in round one were identified (10 female and 16 male). The median age 